\title{
Existence and Uniqueness Results for a Class of Singular Fractional Boundary Value Problems with the $p$-Laplacian Operator via the Upper and Lower Solutions Approach
}

\author{
KumSong Jong ${ }^{(D}$, HuiChol Choi, KyongJun Jang, and SunAe Pak \\ Faculty of Mathematics, Kim Il Sung University, Pyongyang, Democratic People's Republic of Korea \\ Correspondence should be addressed to KumSong Jong; ksjong1016@163.com
}

Received 2 May 2020; Accepted 1 June 2020; Published 15 June 2020

Academic Editor: Adrian Petrusel

Copyright (C) 2020 KumSong Jong et al. This is an open access article distributed under the Creative Commons Attribution License, which permits unrestricted use, distribution, and reproduction in any medium, provided the original work is properly cited.

\begin{abstract}
In this paper, we study the existence and uniqueness of positive solutions to a class of multipoint boundary value problems for singular fractional differential equations with the $p$-Laplacian operator. Here, the nonlinear source term $f$ permits singularity with respect to its time variable $t$. Some fixed-point theorems such as the Leray-Schauder nonlinear alternative, the Schauder fixed-point theorem, and the Banach contraction mapping principle and the properties of the Gauss hypergeometric function are used to prove our main results. And by employing the upper and lower solutions technique, we derive a new approach to obtain the maximal and minimal solutions to the given problem. Finally, we present some examples to demonstrate our existence and uniqueness results.
\end{abstract}

\section{Introduction}

In this paper, we consider the existence of positive solutions of the following $m$-point boundary value problems for singular nonlinear fractional differential equations

$$
\left\{\begin{array}{l}
D_{0+}^{\beta}\left(\varphi_{p}\left(D_{0+}^{\alpha} x(t)\right)\right)=f(t, x(t)), 0<t<1, \\
x(0)=0, D_{0+}^{\gamma} x(1)=\sum_{i=1}^{m-2} \xi_{i} D_{0+}^{\gamma} x\left(\eta_{i}\right), \\
D_{0+}^{\alpha} x(0)=0, \varphi_{p}\left(D_{0+}^{\alpha} x(1)\right)=\sum_{i=1}^{m-2} \zeta_{i} \varphi_{p}\left(D_{0+}^{\alpha} x\left(\eta_{i}\right)\right),
\end{array}\right.
$$

where $1<\alpha, \beta \leq 2,0<\gamma<1,0<\xi_{i}, \eta_{i}, \zeta_{i}<1, i=1,2, \cdots, m-$ 2 , and $D_{0+}^{\alpha}, D_{0+}^{\beta}, D_{0+}^{\gamma}$ are the standard Riemann-Liouville derivatives. Here, $f \in C((0,1) \times[0,+\infty),[0,+\infty)), f(t, x)$ is singular at $t=0,1$ (i.e., $\lim _{t \rightarrow 0+} f(t, \cdot)=+\infty$, $\lim _{t \rightarrow 1-} f(t, \cdot)=+\infty$ ) and $\varphi_{p}$ is defined as $\varphi_{p}(s)=|s|^{p-2} s, p>1$.

Fractional calculus has a history of several hundred years, and many valuable results, which have contributed to the development of mathematical theories and their application to practice, have been created during its historical process (see [1]). Also, fractional differential equations are one of the powerful tools to model and solve scientific and technological problems arising in physics, chemistry, biology, and mechanics, and it has developed more and more in depth (see [2]). In particular, after Leibenson's work dealt with the application of the integer-order differential equation with the $p$-Laplacian operator to the analysis of turbulent flow in porous media (see [3]), many valuable existence results for this equation have been achieved, and recently, the achievements obtained in this integer-order differential equation are more generalized to the fractional differential equation (see [4-6] and the references therein). However, due to the nonlinearity of the $p$-Laplacian operator, not much has been studied on the solutions to singular fractional differential equations with this operator and many researchers have been paid their attention to those equations. For instance, by using the fixed-point theorem of mixed monotone operators, Jong et al. [7] proved the existence of positive solutions to the boundary value problem (1), in which the nonlinear source term $f(t, x)$ was singular with respect to its space variable $x$, and proposed a new approach by which the approximate 
solution of the given problem could be obtained. Unlikely in [7], this paper deals with the boundary value problem (1), in which the function $f(t, x)$ permits singularity with respect to its time variable $t$.

Many researchers have derived some important results for solutions to boundary value problems of fractional differential equations with singularity with respect to the time variable (see [8-29]). In [14], Henderson et al. established the existence and multiplicity of positive solutions for a system of nonlinear Riemann-Liouville fractional differential equations

$$
\left\{\begin{array}{l}
D_{0+}^{\alpha} u(t)+f(t, u(t), v(t))=0, t \in(0,1) \\
D_{0+}^{\beta} u(t)+g(t, u(t), v(t))=0, t \in(0,1)
\end{array}\right.
$$

with the coupled multipoint boundary conditions

$$
\left\{\begin{array}{l}
u(0)=u^{\prime}(0)=\cdots=u^{(n-2)}(0)=0,\left.D_{0+}^{p_{1}} u(t)\right|_{t=1}=\left.\sum_{i=1}^{N} a_{i} D_{0+}^{q_{1}} v(t)\right|_{t=\xi_{i}}, \\
v(0)=v^{\prime}(0)=\cdots=v^{(m-2)}(0)=0,\left.D_{0+}^{p_{2}} v(t)\right|_{t=1}=\left.\sum_{i=1}^{M} b_{i} D_{0+}^{q_{2}} u(t)\right|_{t=\eta_{i}},
\end{array}\right.
$$

where $\alpha \in(n-1, n], \beta \in(m-1, m]$ and the functions $f, g$ can be singular at the points $t=0$ and/or $t=1$. They employed the Guo-Krasnosel'skii fixed-point theorem to prove that their problem has at least one positive solution. And $\mathrm{Wu}$ and Zhou [6] used the upper and lower solutions method to study the existence of positive solutions for the fractional-order eigenvalue problem with the $p$-Laplacian operator

$$
\left\{\begin{array}{l}
-D_{t}^{\beta}\left(\varphi_{p}\left(D_{t}^{\alpha} x\right)\right)(t)=\lambda f(t, x(t)), t \in(0,1) \\
x(0)=0, D_{t}^{\alpha} x(0)=0 \\
D_{t}^{\gamma} x(1)=\sum_{j=1}^{m-2} a_{j} D_{t}^{\gamma} x\left(\xi_{j}\right)
\end{array}\right.
$$

where $1<\alpha \leq 2,0<\beta, \gamma \leq 1$ and $f$ can be singular at $t=0,1$ and $x=0$ (for more detailed information about the upper and lower solutions method to solve integral and differential equations, see [30]).

Taking the previous results together, to our best knowledge, very little is known about the existence and uniqueness of positive solutions of $p$-Laplacian fractional boundary value problems with singularities with respect to their time variable.

Motivated by the above works, in this paper, we first apply the Leray-Schauder nonlinear alternative to establish the existence of solutions to our problem (1) and then use the Schauder fixed-point theorem and upper and lower control functions to derive the upper and lower solutions method to obtain the maximal and minimal solutions. And we prove the uniqueness of solutions to the given problem by using some useful properties of the Gauss hypergeometric function ${ }_{2} F_{1}(a, b, c, s)$ and the Banach contraction mapping principle.

Throughout this paper, we suppose that

$$
3<\alpha+\beta \leq 4, \alpha-\gamma-1>0, \sum_{i=1}^{m-2} \xi_{i} \eta_{i}^{\alpha-\gamma-1}<1, \sum_{i=1}^{m-2} \zeta_{i} \eta_{i}^{\beta-1}<1
$$

\section{Preliminaries}

For the convenience of the readers, we will give some necessary definitions and lemmas here.

The Riemann-Liouville fractional integral and the Riemann-Liouville fractional derivative of order $\alpha>0$ of a function $f:(0, \infty) \longrightarrow \mathbf{R}$ are given by

$$
\begin{aligned}
\left(I_{0+}^{\alpha} f\right)(t) & :=\frac{1}{\Gamma(\alpha)} \int_{0}^{t}(t-s)^{\alpha-1} f(s) d s,\left(D_{0+}^{\alpha} f\right)(t) \\
& :=\frac{1}{\Gamma(n-\alpha)}\left(\frac{d}{d t}\right)^{n} \int_{0}^{t}(t-s)^{n-\alpha-1} f(s) d s
\end{aligned}
$$

where $n=[\alpha]+1$, provided that the right-hand sides are pointwise defined on $(0, \infty)$ (see [1]).

Lemma 1 (see [31]). Assume that $u \in C(0,1) \cap L(0,1)$ with a fractional derivative of order $\alpha>0$ that belongs to $C(0,1) \cap$ $L(0,1)$. Then,

$$
I_{0+}^{\alpha} D_{0+}^{\alpha} u(t)=u(t)+C_{1} t^{\alpha-1}+C_{2} t^{\alpha-2}+\cdots+C_{N} t^{\alpha-N},
$$

for some $C_{i} \in \mathbf{R}, i=1,2, \cdots, N$, where $N$ is the smallest integer greater than or equal to $\alpha$.

Lemma 2 (see [32]). (Schauder fixed-point theorem). Let D be a nonempty, closed, bounded, and convex subset of a Banach space $X$, and suppose $A: D \longrightarrow D$ is a compact operator. Then, $A$ has a fixed point.

Lemma 3 (see [33]). Let $X$ be a Banach space with D a closed and convex subset of $X$. Assume $U$ is a relatively open subset of $D$, with $0 \in U$, and $A: \bar{U} \longrightarrow D$ is a compact map. Then, either,

(i) A has a fixed point in $\bar{U}$, or

(ii) there is a point $x \in \partial U$ and $\lambda \in(0,1)$, with $x=\lambda A x$

Lemma 4. Let $z:(0,1) \longrightarrow[0,+\infty)$ be a continuous function such that

$\exists \sigma_{0}, \sigma_{1} \in(0,1) ; \lim _{t \rightarrow 0+} t^{\sigma_{0}} z(t)=z_{0}>0, \lim _{t \rightarrow 1-}(1-t)^{\sigma_{1}} z(t)=z_{1}>0$, 
and define the function $K$ as

$$
K(t):= \begin{cases}0, & t=0, \\ \int_{0}^{t}(t-s)^{\beta-1} z(s) d s, & t \in(0,1] .\end{cases}
$$

Then, $K$ is continuous on $[0,1]$.

Proof. From the assumption (5), we can see easily that

$$
\begin{aligned}
& \exists \delta_{0}>0 ; \forall t\left(0<t<\delta_{0}\right), \frac{1}{2} z_{0}<t^{\sigma_{0}} z(t)<\frac{3}{2} z_{0}, \\
& \exists \delta_{1}>0 ; \forall t\left(1-\delta_{1}<t<1\right), \frac{1}{2} z_{1}<(1-t)^{\sigma_{1}} z(t)<\frac{3}{2} z_{1} .
\end{aligned}
$$

Put as follows

$$
\delta:=\min \left\{\frac{1}{4}, \delta_{0}, \delta_{1}\right\}, M_{z}:=\max _{\delta \leq t \leq 1-\delta}|z(t)|
$$

Then, we divide the proof of this lemma into the following three cases:

If $t \in(0, \delta]$, the definition of an improper integral

$$
K(t)=\lim _{t^{\prime} \rightarrow 0+} \int_{t^{\prime}}^{t}(t-s)^{\beta-1} z(s) d s
$$

yields

$$
\begin{aligned}
\int_{t^{\prime}}^{t}(t-s)^{\beta-1} z(s) d s & =\int_{t^{\prime}}^{t}(t-s)^{\beta-1} s^{-\sigma_{0}} s^{\sigma_{0}} z(s) d s \\
& \leq \frac{3}{2} z_{0} \int_{t^{\prime}}^{t}(t-s)^{\beta-1} s^{-\sigma_{0}} d s \\
& \leq \frac{3}{2} z_{0} \int_{0}^{1}(1-s)^{\beta-1} s^{-\sigma_{0}} t^{\beta-\sigma_{0}} d s \\
& =\frac{3}{2} z_{0} B\left(\beta, 1-\sigma_{0}\right) t^{\beta-\sigma_{0}} .
\end{aligned}
$$

Take the limit $t^{\prime} \longrightarrow 0+$ to obtain

$$
K(t) \leq \frac{3}{2} z_{0} B\left(\beta, 1-\sigma_{0}\right) t^{\beta-\sigma_{0}} .
$$

This implies $\lim _{t \rightarrow 0+} K(t)=0=K(0)$.

If $t \in(\delta, 1-\delta]$, we can get

$$
K(t)=\lim _{t^{\prime} \rightarrow 0+} \int_{t^{\prime}}^{\delta}(t-s)^{\beta-1} z(s) d s+\int_{\delta}^{t}(t-s)^{\beta-1} z(s) d s .
$$

In a similar way above, the first term of the right side in Equation (17) can be evaluated as

$$
\lim _{t^{\prime} \rightarrow 0+} \int_{t^{\prime}}^{\delta}(t-s)^{\beta-1} z(s) d s \leq \frac{3}{2} z_{0} B\left(\beta, 1-\sigma_{0}\right) t^{\beta-\sigma_{0}} .
$$

And for the second term of the right side in Equation (17), it can be easily seen that

$$
\begin{aligned}
\int_{\delta}^{t}(t-s)^{\beta-1} z(s) d s & \leq M_{z} \int_{\delta}^{t}(t-s)^{\beta-1} d s \\
& \leq M_{z} \int_{0}^{t}(t-s)^{\beta-1} d s=\frac{1}{\beta} M_{z} t^{\beta}
\end{aligned}
$$

Combining these two inequalities above, we can find

$$
K(t) \leq \frac{3}{2} z_{0} B\left(\beta, 1-\sigma_{0}\right) t^{\beta-\sigma_{0}}+\frac{1}{\beta} M_{z} t^{\beta} .
$$

For the case $t \in(1-\delta, 1]$, if $t<1$, then we have

$$
\begin{aligned}
K(t)= & \lim _{t^{\prime} \rightarrow 0+} \int_{t^{\prime}}^{\delta}(t-s)^{\beta-1} z(s) d s+\int_{\delta}^{1-\delta}(t-s)^{\beta-1} z(s) d s \\
& +\int_{1-\delta}^{t}(t-s)^{\beta-1} z(s) d s .
\end{aligned}
$$

Similarly to that given above, we obtain

$$
\begin{aligned}
& \lim _{t^{\prime} \rightarrow 0+} \int_{t^{\prime}}^{\delta}(t-s)^{\beta-1} z(s) d s \\
& \quad \leq \frac{3}{2} z_{0} B\left(\beta, 1-\sigma_{0}\right) t^{\beta-\sigma_{0}}, \int_{\delta}^{1-\delta}(t-s)^{\beta-1} z(s) d s \leq \frac{1}{\beta} M_{z} t^{\beta} .
\end{aligned}
$$

By simple calculation, we can get

$$
\begin{aligned}
\int_{1-\delta}^{t}(t-s)^{\beta-1} z(s) d s & =\int_{1-\delta}^{t}(t-s)^{\beta-1}(1-s)^{-\sigma_{1}}(1-s)^{\sigma_{1}} z(s) d s \\
& \leq \frac{3}{2} z_{1} \int_{1-\delta}^{t}(t-s)^{\beta-1}(1-s)^{-\sigma_{1}} d s \\
& \leq \frac{3}{2} z_{1} \int_{0}^{1}(1-s)^{\beta-1}(1-s)^{-\sigma_{1}} d s \\
& =\frac{3}{2\left(\beta-\sigma_{1}\right)} z_{1} .
\end{aligned}
$$

So for any $t \in(1-\delta, 1)$, it holds that

$$
K(t) \leq \frac{3}{2} z_{0} B\left(\beta, 1-\sigma_{0}\right) t^{\beta-\sigma_{0}}+\frac{1}{\beta} M_{z} t^{\beta}+\frac{3}{2\left(\beta-\sigma_{1}\right)} z_{1} .
$$

If $t=1$, the definition of an improper integral also implies $K(1)=\lim _{t \rightarrow 1-} K(t)$. Taking the limit $t \longrightarrow 1-$ on both sides of the inequality (11), we can obtain

$$
K(1) \leq \frac{3}{2} z_{0} B\left(\beta, 1-\sigma_{0}\right)+\frac{1}{\beta} M_{z}+\frac{3}{2\left(\beta-\sigma_{1}\right)} z_{1}<+\infty .
$$

Therefore, it follows directly from the inequalities (7) and (10), (11), (12), (13) that $K$ is well-defined on $[0,1]$. Since $z$ 
$\in C(0,1)$, it is obvious that $K$ is continuous on $(0,1)$. Combining this with the continuity of $K$ at $t=0,1$, we can prove that $K$ is a continuous function on $[0,1]$.

Lemma 5. Let $z:(0,1) \longrightarrow[0,+\infty)$ be a continuous function such that satisfies (6). Then, the boundary value problem

$$
\left\{\begin{array}{l}
D_{0+}^{\beta} v(t)+z(t)=0 \\
v(0)=0, v(1)=\sum_{i=1}^{m-2} \zeta_{i} v\left(\eta_{i}\right)
\end{array}\right.
$$

has a unique solution which is given by

$$
v(t)=\int_{0}^{1} H(t, s) z(s) d s
$$

where

$$
H(t, s)=H_{1}(t, s)+H_{2}(t, s)
$$

in which

$$
\begin{aligned}
H_{1}(t, s)= & \frac{1}{\Gamma(\beta)}\left\{\begin{array}{l}
t^{\beta-1}(1-s)^{\beta-1}-(t-s)^{\beta-1}, 0 \leq s \leq t \leq 1, \\
t^{\beta-1}(1-s)^{\beta-1}, 0 \leq t \leq s \leq 1,
\end{array}\right. \\
H_{2}(t, s)= & \frac{t^{\beta-1}}{B \Gamma(\beta)}\left[\sum_{s<\eta_{i}} \zeta_{i}\left[\eta_{i}^{\beta-1}(1-s)^{\beta-1}-\left(\eta_{i}-s\right)^{\beta-1}\right]\right. \\
& \left.+\sum_{s \geq \eta_{i}} \zeta_{i} \eta_{i}^{\beta-1}(1-s)^{\beta-1}\right], t, s \in[0,1],
\end{aligned}
$$

where

$$
B=1-\sum_{i=1}^{m-2} \zeta_{i} \eta_{i}^{\beta-1}
$$

Remark 6. In Lemma 5, a function $v \in C[0,1]$ with a fractional derivative of order $\beta$ that belongs to $C(0,1)$ (i.e., $\left.D_{0+}^{\beta} u \in C(0,1)\right)$ is said to be a solution of the boundary value problem (14) if it satisfies the fractional differential equation and the boundary conditions of (12).

Proof. As we can see in the proof of Lemma 4, we have

$$
\begin{aligned}
\int_{0}^{1} t^{\sigma_{0}}(1-t)^{\sigma_{1}} z(t) d t & <+\infty, \int_{0}^{1} t^{-\sigma_{0}}(1-t)^{-\sigma_{1}} d t \\
& =B\left(1-\sigma_{0}, 1-\sigma_{1}\right)<+\infty
\end{aligned}
$$

So, we can get

$\int_{0}^{1} z(t) d t=\int_{0}^{1} t^{-\sigma_{0}}(1-t)^{-\sigma_{1}} t^{\sigma_{0}}(1-t)^{\sigma_{1}} z(t) d t<+\infty$

This implies $z \in L(0,1)$.

Also, Lemma 4 asserts that

$$
I_{0+}^{\beta} z(t)=\frac{1}{\Gamma(\beta)} \int_{0}^{t}(t-s)^{\beta-1} z(s) d s \in C[0,1] .
$$

Since $z \in C(0,1) \cap L(0,1)$ and $I_{0+}^{\beta} z \in C[0,1]$, it follows from Lemma 1 that a solution of the boundary value problem (14), $v \in C[0,1]$, satisfies

$$
v(t)+c_{1} t^{\beta-1}+c_{2} t^{\beta-2}=-I_{0+}^{\beta} z(t) .
$$

For the rest of the proof, it is easy to see that doing as in the proof of Lemma 4 in [12] leads to a conclusion of this lemma.

Lemma 7 (see [4]). If $\sum_{i=1}^{m-2} \zeta_{i} \eta_{i}^{\beta-1}<1$, then the function $H(t, s)$ in Lemma 5 satisfies the following conditions:

(i) $H(t, s)>0$, for $t, s \in(0,1)$

(ii) $H(t, s) \leq H_{*}(s, s)$, for $t, s \in[0,1]$

where

$$
H_{*}(s, s)=\frac{1}{B \Gamma(\beta)}(1-s)^{\beta-1}
$$

As we can see in the proof of Lemma 4 in [4], it holds that

$$
\begin{aligned}
v(t) & =\int_{0}^{1} H(t, s) z(s) d s \\
& =-I_{0+}^{\beta} z(t)-\frac{1}{B}\left[\left.\sum_{i=1}^{m-2} \zeta_{i} I_{0+}^{\beta} z(t)\right|_{t=\eta_{i}}-\left.I_{0+}^{\beta} z(t)\right|_{t=1}\right] t^{\beta-1} .
\end{aligned}
$$

Lemma 8 (see [34]). Let $y \in C[0,1]$. Then, the boundary value problem

$$
\left\{\begin{array}{l}
D_{0+}^{\alpha} u(t)+y(t)=0,0<t<1, \\
u(0)=0, D_{0+}^{\gamma} u(1)=\sum_{i=1}^{m-2} \xi_{i} D_{0+}^{\gamma} u\left(\eta_{i}\right),
\end{array}\right.
$$

has a unique solution which is given by

$$
u(t)=\int_{0}^{1} G(t, s) y(s) d s
$$


where

$$
G(t, s)=G_{1}(t, s)+G_{2}(t, s)
$$

in which

$$
\begin{aligned}
G_{1}(t, s)= & \left\{\begin{array}{l}
\frac{t^{\alpha-1}(1-s)^{\alpha-\gamma-1}-(t-s)^{\alpha-1}}{\Gamma(\alpha)}, \quad 0 \leq s \leq t \leq 1, \\
\frac{t^{\alpha-1}(1-s)^{\alpha-\gamma-1}}{\Gamma(\alpha)}, \quad 0 \leq t \leq s \leq 1,
\end{array}\right. \\
G_{2}(t, s)= & \frac{t^{\alpha-1}}{A \Gamma(\alpha)}\left[\sum_{s<\eta_{i}} \xi_{i}\left[\eta_{i}^{\alpha-\gamma-1}(1-s)^{\alpha-\gamma-1}-\left(\eta_{i}-s\right)^{\alpha-\gamma-1}\right]\right. \\
& \left.+\sum_{s \geq \eta_{i}} \xi_{i} \eta_{i}^{\alpha-\gamma-1}(1-s)^{\alpha-\gamma-1}\right], t, s \in[0,1],
\end{aligned}
$$

where

$$
A=1-\sum_{i=1}^{m-2} \xi_{i} \eta_{i}^{\alpha-\gamma-1}
$$

Lemma 9 (see [34]). If $\sum_{i=1}^{m-2} \xi_{i} \eta_{i}^{\alpha-\gamma-1}<1$, then the function $G(t, s)$ in Lemma 8 satisfies the following conditions:

(i) $G(t, s)>0$, for $t, s \in(0,1)$

(ii) $G(t, s) \leq G_{*}(s, s)$, for $t, s \in[0,1]$

where

$$
G_{*}(s, s)=\frac{1}{A \Gamma(\alpha)}(1-s)^{\alpha-\gamma-1}
$$

The following useful properties of $\varphi_{p}(\cdot)$ which will be used later can be found in [5]:

(i) If $1<p<2, x y>0$, and $|x|,|y| \geq m>0$, then

$$
\left|\varphi_{p}(x)-\varphi_{p}(y)\right| \leq(p-1) m^{p-2}|x-y|
$$

(ii) If $p>2,|x|,|y| \leq M$, then

$$
\left|\varphi_{p}(x)-\varphi_{p}(y)\right| \leq(p-1) M^{p-2}|x-y| .
$$

\section{Main Results}

In this section, we will prove the existence and uniqueness of positive solutions for the boundary value problem (1) and derive the upper and lower solutions method by using some fixed-point theorems.

\subsection{The Existence Results for Problem (1)}

Definition 10. A function $x \in\left\{u \mid u \in C[0,1], D_{0+}^{\alpha} u \in C[0,1]\right.$, $\left.D_{0+}^{\beta}\left(\phi_{p}\left(D_{0+}^{\alpha} u\right)\right) \in C(0,1)\right\}$ is called a solution of problem (1) if it satisfies the fractional differential equation and the boundary conditions of (1).

The following hypothesis concerned with the function $f$ $(t, x)$, which permits singularity with respect to time variable, will be used in this article.

(H1). There exist $\sigma_{0}, \sigma_{1} \in(0,1)$ such that for any $x \in[0,+\infty)$,

$$
\lim _{t \rightarrow 0+} t^{\sigma_{0}} f(t, x)=f_{0}(x)>0, \lim _{t \rightarrow 1-}(1-t)^{\sigma_{1}} f(t, x)=f_{1}(x)>0 .
$$

Let $E$ be the Banach space $C[0,1]$ equipped with the norm $\|u\|=\max _{0 \leq t \leq 1}|u(t)|$ and put $P:=\{u \in E \mid u(t) \geq 0, t \in[0,1]\}$.

Lemma 11. Assume that the hypothesis (H1) holds. Then, the function $x \in P$ is a solution of the problem (1) if and only if $x$ is a solution of the integral equation

$$
x(t)=\int_{0}^{1} G(t, s) \varphi_{q}\left(\int_{0}^{1} H(s, \tau) f(\tau, x(\tau)) d \tau\right) d s
$$

in $P$, where $q$ is a real number such that $(1 / p)+(1 / q)=1$.

Proof. Suppose that $x \in P$ is a solution of the problem (1). Putting $w(t):=D_{0+}^{\alpha} x(t)$, by using Lemma 8 , we have

$$
x(t)=-\int_{0}^{1} G(t, s) w(s) d s .
$$

Also putting $v(t):=\varphi_{p}(w(t))$, by Lemma 5 , we obtain

$$
v(t)=-\int_{0}^{1} H(t, s) f(s, x(s)) d s .
$$

Since $\varphi_{p}^{-1}=\varphi_{q}$, Equations (47) and(48) yields

$$
x(t)=\int_{0}^{1} G(t, s) \varphi_{q}\left(\int_{0}^{1} H(s, \tau) f(\tau, x(\tau)) d \tau\right) d s .
$$

It is obvious that $x \in P$ and $x$ is a solution of the integral Equation (46).

Conversely, suppose that $x \in P$ is a solution of the integral Equation (46). Put as follows:

$$
z(t):=f(t, x(t)), v(t):=\varphi_{q}\left(\int_{0}^{1} H(t, s) z(s) d s\right)
$$


Then, Equation (36) implies that $v \in C[0,1]$. From the definition of $G(t, s)$, we also have

$$
\begin{aligned}
& x(t)= \int_{0}^{1} G(t, s) v(s) d s \\
&=-I_{0+}^{\alpha} v(t)-\frac{\Gamma(\alpha-\gamma)}{A \Gamma(\alpha)}\left[\left.\sum_{i=1}^{m-2} \xi_{i} I_{0+}^{\alpha-\gamma} v(t)\right|_{t=\eta_{i}}\right. \\
&\left.\quad-\left.I_{0+}^{\alpha-\gamma} v(t)\right|_{t=1}\right] t^{\alpha-1} .
\end{aligned}
$$

So, we can get

$$
D_{0+}^{\alpha} x(t)=-v(t)
$$

and it follows that $D_{0+}^{\alpha} x \in C[0,1]$. Combining Equation (36) with

$$
\begin{aligned}
D_{0+}^{\beta}\left(\varphi_{p}\left(D_{0+}^{\alpha} x(t)\right)\right) & =D_{0+}^{\beta}\left(\varphi_{p}(-v(t))\right) \\
& =D_{0+}^{\beta}\left(-\int_{0}^{1} H(t, s) z(s) d s\right),
\end{aligned}
$$

we can see

$$
D_{0+}^{\beta}\left(\varphi_{p}\left(D_{0+}^{\alpha} x(t)\right)\right)=z(t)
$$

This yields $D_{0+}^{\beta}\left(\varphi_{p}\left(D_{0+}^{\alpha} x\right)\right) \in C(0,1)$.

Since the boundary value problems (14) and (16) have unique solutions by Lemma 5 and Lemma 8 , we can find that the function $x$ satisfies the fractional differential equation and the boundary conditions of the problem (1). This completes the proof.

Define the operator $T$ on $P$ as follows:

$$
T x(t):=\int_{0}^{1} G(t, s) \varphi_{q}\left(\int_{0}^{1} H(s, \tau) f(\tau, x(\tau)) d \tau\right) d s .
$$

Then, the function $x \in P$ is a solution of the integral Equation (46) if and only if the operator $T$ has a fixed point $x$ in $P$.

Lemma 12. If the hypothesis (H1) holds, then

$$
T(P) \subset P \text {. }
$$

Proof. The conclusion of this lemma easily follows from Lemma 4, so we omit the details.
For convenience we define the function $F(t, x)$ on $[0,1]$ $\times[0,+\infty)$ as follows:

$$
F(t, x):= \begin{cases}f_{0}(x), & t=0 \wedge \forall x \in[0,+\infty), \\ t^{\sigma_{0}}(1-t)^{\sigma_{1}} f(t, x), & \forall(t, x) \in(0,1) \times[0,+\infty), \\ f_{1}(x), & t=1 \wedge \forall x \in[0,+\infty) .\end{cases}
$$

Obviously, we can see that $F \in C([0,1] \times[0,+\infty))$. In fact, using the hypothesis (H1), for any $x \in[0,+\infty)$, it holds that

$$
\begin{aligned}
\lim _{t \rightarrow 0+} F(t, x) & =\lim _{t \rightarrow 0+} t^{\sigma_{0}}(1-t)^{\sigma_{1}} f(t, x)=\lim _{t \rightarrow 0+} t^{\sigma_{0}} f(t, x) \\
& =f_{0}(x)=F(0, x), \\
\lim _{t \rightarrow 1-} F(t, x) & =\lim _{t \rightarrow 1-} t^{\sigma_{0}}(1-t)^{\sigma_{1}} f(t, x) \\
& =\lim _{t \rightarrow 1-}(1-t)^{\sigma_{1}} f(t, x)=f_{1}(x)=F(1, x),
\end{aligned}
$$

and $F \in C((0,1) \times[0,+\infty))$.

Lemma 13. If the hypothesis (H1) holds, then the operator $T: P \longrightarrow P$ is completely continuous.

Proof. We first prove that the operator $T$ is continuous on $P$. For this, choose any $x \in P$ and any sequence $\left\{x_{n}\right\}_{n=1}^{\infty} \subset P$ convergent to $x$. Then, there exists $L_{1}>0$ such that $\forall n,\left\|x_{n}\right\| \leq L_{1}$. From the continuity of the function $F$, we can put

$$
M_{1}:=\max _{\substack{0 \leq t \leq 1 \\ 0 \leq x \leq L_{1}}}|F(t, x)|
$$

From the definition of the function $F$, we can see that for any $0<t<1$,

$$
\left|f\left(t, x_{n}(t)\right)\right| \leq t^{-\sigma_{0}}(1-t)^{-\sigma_{1}} M_{1} \text {. }
$$

Since $x_{n} \in C[0,1]$, we have

$$
\lim _{n \rightarrow \infty} f\left(t, x_{n}(t)\right)=f(t, x(t)) .
$$

A simple calculation provides that

$$
\int_{0}^{1} t^{-\sigma_{0}}(1-t)^{-\sigma_{1}} M_{1} d t=M_{1} \cdot B\left(1-\sigma_{0}, 1-\sigma_{1}\right)<+\infty,
$$

and therefore, by using the Lebesgue dominated convergence theorem, we know

$$
\lim _{n \rightarrow \infty} \int_{0}^{1} f\left(t, x_{n}(t)\right) d t=\int_{0}^{1} f(t, x(t)) d t
$$


Combining this with $H \in C([0,1] \times[0,1])$, we can obtain that for any $s \in[0,1]$,

$$
\begin{array}{r}
\lim _{n \rightarrow \infty} \varphi_{q}\left(\int_{0}^{1} H(s, \tau) f\left(\tau, x_{n}(\tau)\right) d \tau\right) \\
\quad=\varphi_{q}\left(\int_{0}^{1} H(s, \tau) f(\tau, x(\tau)) d \tau\right) .
\end{array}
$$

Since $G \in C([0,1] \times[0,1])$, we can get that for any $t \in$ $[0,1]$,

$$
\begin{array}{r}
\lim _{n \rightarrow \infty} \int_{0}^{1} G(t, s) \varphi_{q}\left(\int_{0}^{1} H(s, \tau) f\left(\tau, x_{n}(\tau)\right) d \tau\right) d s \\
\quad=\int_{0}^{1} G(t, s) \varphi_{q}\left(\int_{0}^{1} H(s, \tau) f(\tau, x(\tau)) d \tau\right) d s .
\end{array}
$$

This gives

$$
\forall t \in[0,1], \lim _{n \rightarrow \infty} T x_{n}(t)=T x(t) .
$$

Next, we show that for any bounded set $\Omega \subset P, T(\Omega)$ is relatively compact. To do this, by the Arzela-Ascoli theorem, it is sufficient to prove that $T(\Omega)$ is uniformly bounded and equicontinuous. Denote $\Omega=\left\{u \in P:\|u\| \leq L_{2}\right\}$.

From Lemma 7 and Lemma 9, we see that for any $x \in \Omega$ and any $t \in[0,1]$,

$$
\begin{aligned}
|T x(t)| & =\int_{0}^{1} G(t, s) \varphi_{q}\left(\int_{0}^{1} H(s, \tau) f(\tau, x(\tau)) d \tau\right) d s \\
& \leq \int_{0}^{1} G_{*}(s, s) d s \cdot \varphi_{q}\left(\int_{0}^{1} H_{*}(\tau, \tau) f(\tau, x(\tau)) d \tau\right) .
\end{aligned}
$$

Put $M_{2}:=\max _{\substack{0 \leq t \leq 1 \\ 0 \leq x \leq L_{2}}}|F(t, x)|$. Since $\|x\| \leq L_{2}$, we have

$$
\begin{aligned}
& \int_{0}^{1} H_{*}(\tau, \tau) f(\tau, x(\tau)) d \tau \\
& \quad=\int_{0}^{1} H_{*}(\tau, \tau) \tau^{-\sigma_{0}}(1-\tau)^{-\sigma_{1}} F(\tau, x(\tau)) d \tau \\
& \quad \leq \frac{M_{2}}{B \Gamma(\beta)} \int_{0}^{1}(1-\tau)^{\beta-1} \cdot \tau^{-\sigma_{0}}(1-\tau)^{-\sigma_{1}} d \tau \\
& \quad=\frac{M_{2}}{B \Gamma(\beta)} \cdot B\left(1-\sigma_{0}, \beta-\sigma_{1}\right)
\end{aligned}
$$

Therefore, we know

$$
|T u(t)| \leq \frac{1}{A(\alpha-\gamma) \Gamma(\alpha)} \varphi_{q}\left(\frac{M_{2} \cdot B\left(1-\sigma_{0}, \beta-\sigma_{1}\right)}{B \Gamma(\beta)}\right) .
$$

By using the inequality (20), we can get that for any $t_{1}$, $t_{2} \in[0,1]$,

$$
\begin{aligned}
\mid T x & \left(t_{2}\right)-T x\left(t_{1}\right) \mid \\
& =\left|\int_{0}^{1}\left[G\left(t_{2}, s\right)-G\left(t_{1}, s\right)\right] \varphi_{q}\left(\int_{0}^{1} H(s, \tau) f(\tau, x(\tau)) d \tau\right) d s\right| \\
& \leq \int_{0}^{1}\left|G\left(t_{2}, s\right)-G\left(t_{1}, s\right)\right| \varphi_{q}\left(\int_{0}^{1} H_{*}(\tau, \tau) f(\tau, x(\tau)) d \tau\right) d s \\
& \leq \varphi_{q}\left(\frac{M_{2} \cdot B\left(1-\sigma_{0}, \beta-\sigma_{1}\right)}{B \Gamma(\beta)}\right) \int_{0}^{1}\left|G\left(t_{2}, s\right)-G\left(t_{1}, s\right)\right| d s .
\end{aligned}
$$

Combining this inequality with the uniform continuity of $G(t, s)$ on $[0,1] \times[0,1]$, it holds that for any $\varepsilon>0$, there exists $\delta>0$ such that

$\forall t_{1}, t_{2} \in[0,1]\left(\left|t_{2}-t_{1}\right|<\delta\right), \forall s \in[0,1],\left|T x\left(t_{2}\right)-T x\left(t_{1}\right)\right|<\varepsilon$.

This implies that $T(\Omega)$ is equicontinuous. The proof is completed.

Put $\bar{M}=[(\alpha-\gamma) A \Gamma(\alpha)]^{p-1} B \Gamma(\beta)$ and list more hypotheses to be used in this paper.

(H2). There exists a nondecreasing function $g \in C([0,+\infty)$, $[0,+\infty))$ such that $t^{\sigma_{0}}(1-t)^{\sigma_{1}} f(t, x) \leq g(x)$ for any $(t, x) \epsilon$ $(0,1) \times[0,+\infty)$.

(H3). There exists $R>0$ such that $R^{p-1} / g(R) \geq B\left(1-\sigma_{0}, \beta-\right.$ $\left.\sigma_{1}\right) / \bar{M}$.

Theorem 14. Assume that the hypotheses (H1)-(H3) hold. Then, the problem (1) has at least one solution in $B_{R}$, where $B_{R}=\{u \in P \mid\|u\| \leq R\}$.

Proof. Putting $U_{R}=\{u \in P \mid\|u\|<R\}$, it is obvious that $\bar{U}_{R}$ $=B_{R}$. By using Lemma 13, we can know that the operator $T: \bar{U}_{R} \longrightarrow P$ is completely continuous.

Assume that there exist a point $x \in \partial U_{R}$ and a number $\lambda \in(0,1)$, with $x=\lambda T x$. By the hypothesis (H2), we see that for any $t \in[0,1]$,

$$
\begin{aligned}
x(t) & =\lambda T x(t)=\lambda \int_{0}^{1} G(t, s) \varphi_{q}\left(\int_{0}^{1} H(s, \tau) f(\tau, x(\tau)) d \tau\right) d s \\
& \leq \lambda \int_{0}^{1} G(t, s) \varphi_{q}\left(\int_{0}^{1} H(s, \tau) \tau^{-\sigma_{0}}(1-\tau)^{-\sigma_{1}} g(x(\tau)) d \tau\right) d s \\
& \leq \lambda g(\|x\|)^{q-1} \int_{0}^{1} G(t, s) \varphi_{q}\left(\int_{0}^{1} H(s, \tau) \tau^{-\sigma_{0}}(1-\tau)^{-\sigma_{1}} d \tau\right) d s .
\end{aligned}
$$

This means that $T(\Omega)$ is uniformly bounded. 
Using Lemma 7 and Lemma 9, we have

$$
\begin{aligned}
\int_{0}^{1} G(t, s) \varphi_{q}\left(\int_{0}^{1} H(s, \tau) \tau^{-\sigma_{0}}(1-\tau)^{-\sigma_{1}} d \tau\right) d s \\
\quad \leq \int_{0}^{1} G_{*}(s, s) \varphi_{q}\left(\int_{0}^{1} H_{*}(\tau, \tau) \tau^{-\sigma_{0}}(1-\tau)^{-\sigma_{1}} d \tau\right) d s \\
\quad=\int_{0}^{1} \frac{1}{A \Gamma(\alpha)}(1-s)^{\alpha-\gamma-1} \varphi_{q}\left(\int_{0}^{1} \frac{1}{B \Gamma(\beta)}(1-\tau)^{\beta-1}\right. \\
=\int_{0}^{1} \frac{1}{A \Gamma(\alpha)}(1-s)^{\alpha-\gamma-1}\left(\frac{B\left(1-\sigma_{0}, \beta-\sigma_{1}\right)}{B \Gamma(\beta)}\right)^{q-1} d s \\
=\left(\frac{B\left(1-\sigma_{0}, \beta-\sigma_{1}\right)}{\bar{M}}\right)^{q-1} .
\end{aligned}
$$

Combining these two inequalities, we obtain

$$
\|x\| \leq \lambda g(\|x\|)^{q-1}\left(\frac{B\left(1-\sigma_{0}, \beta-\sigma_{1}\right)}{\bar{M}}\right)^{q-1} .
$$

Now taking $(p-1)$ th power on both sides of the above inequality, since $\|x\|=R,(p-1)(q-1)=1$, and $g$ is a nondecreasing function, we get

$$
R^{p-1} \leq \lambda g(R) \cdot \frac{B\left(1-\sigma_{0}, \beta-\sigma_{1}\right)}{\bar{M}}<g(R) \cdot \frac{B\left(1-\sigma_{0}, \beta-\sigma_{1}\right)}{\bar{M}} .
$$

This is a contradiction to the hypothesis (H3). Therefore, by Lemma 3, the operator $T$ has at least one fixed point in $B_{R}$. The proof is completed.

3.2. Derivation of the Upper and Lower Solutions Method. We define the upper control function $K(t, x)$ and the lower control function $k(t, x)$ on $(0,1) \times[0, R]$ as follows:

$$
K(t, x):=\max _{0 \leq \xi \leq x} f(t, \xi), k(t, x):=\min _{x \leq \xi \leq R} f(t, \xi) .
$$

Then, we know that the functions $K(t, x)$ and $k(t, x)$ are nondecreasing in $x$ and for any $(t, x) \in(0,1) \times[0, R]$,

$$
k(t, x) \leq f(t, x) \leq K(t, x) .
$$

Definition 15. The functions $\bar{x} \in B_{R}$ and $\underline{x} \in B_{R}$ are said to be a upper solution and a lower solution of the integral Equation (46) in $B_{R}$, respectively, if

$$
\begin{aligned}
& \bar{x}(t) \geq \int_{0}^{1} G(t, s) \varphi_{q}\left(\int_{0}^{1} H(s, \tau) K(\tau, \bar{x}(\tau)) d \tau\right) d s, \\
& \underline{x}(t) \leq \int_{0}^{1} G(t, s) \varphi_{q}\left(\int_{0}^{1} H(s, \tau) k(\tau, \underline{x}(\tau)) d \tau\right) d s .
\end{aligned}
$$

Lemma 16. Assume that the hypotheses (H1)-(H3) hold and there exist a upper solution $\bar{x}$ and a lower solution $x$ of the integral Equation (46) in $B_{R}$. Then, the problem (1) has at least one solution in $S$, where $S=\left\{u \in B_{R} \mid \forall t \in[0,1], \underline{x}(t) \leq u(t) \leq\right.$ $\bar{x}(t)\}$.

Proof. It is obvious that $S$ is a nonempty, closed, bounded, and convex subset of the Banach space $E$ and the operator $T: B_{R} \longrightarrow P$ is completely continuous. In a similar way to the proof of the Theorem 14 , for any $x \in B_{R}$, we can get

$$
\forall t \in[0,1],|T x(t)| \leq\left(g(R) \cdot \frac{B\left(1-\sigma_{0}, \beta-\sigma_{1}\right)}{\bar{M}}\right)^{q-1}
$$

By the hypothesis (H3), we have

$$
\forall t \in[0,1],|T x(t)| \leq R
$$

That is, $T\left(B_{R}\right) \subset B_{R}$.

Now we prove $T(S) \subset S$. In fact, since the functions $K(t, x)$ and $k(t, x)$ are nondecreasing in $x$, by using the definition of upper and lower solution and the inequality (21), we obtain

$$
\begin{aligned}
T x(t) & \leq \int_{0}^{1} G(t, s) \varphi_{q}\left(\int_{0}^{1} H(s, \tau) K(\tau, x(\tau)) d \tau\right) d s \\
& \leq \int_{0}^{1} G(t, s) \varphi_{q}\left(\int_{0}^{1} H(s, \tau) K(\tau, \bar{x}(\tau)) d \tau\right) d s \leq \bar{x}(t), \\
T x(t) & \geq \int_{0}^{1} G(t, s) \varphi_{q}\left(\int_{0}^{1} H(s, \tau) k(\tau, x(\tau)) d \tau\right) d s \\
& \geq \int_{0}^{1} G(t, s) \varphi_{q}\left(\int_{0}^{1} H(s, \tau) k(\tau, \underline{x}(\tau)) d \tau\right) d s \geq \underline{x}(t) .
\end{aligned}
$$

Therefore, Lemma 2 assures that the problem (1) has at least one solution in $S$.

Since $F \in C([0,1] \times[0,+\infty))$, we can put

$$
\underline{\rho}:=\min _{\substack{0 \leq t \leq 1 \\ 0 \leq x \leq R}} F(t, x)
$$

Also, put $\bar{\rho}:=g(R)$. Then, by the hypothesis (H2), we know that for any $(t, x) \in(0,1) \times[0, R]$,

$$
\underline{\rho} \leq t^{\sigma_{0}}(1-t)^{\sigma_{1}} f(t, x) \leq \bar{\rho} .
$$

And by using the hypothesis (H3), we have

$$
\bar{\rho} \leq \frac{\bar{M}}{B\left(1-\sigma_{0}, \beta-\sigma_{1}\right)} R^{p-1} .
$$


Denote as follows:

$$
\begin{aligned}
& \underline{\omega}(t):=\underline{\rho}^{q-1} \int_{0}^{1} G(t, s) \varphi_{q}\left(\int_{0}^{1} H(s, \tau) \tau^{-\sigma_{0}}(1-\tau)^{-\sigma_{1}} d \tau\right) d s, \\
& \bar{\omega}(t):=\bar{\rho}^{q-1} \int_{0}^{1} G(t, s) \varphi_{q}\left(\int_{0}^{1} H(s, \tau) \tau^{-\sigma_{0}}(1-\tau)^{-\sigma_{1}} d \tau\right) d s .
\end{aligned}
$$

Theorem 17. If the hypotheses (H1)-(H3) hold, then the problem (1) has at least one solution in $S^{*}$, where $S^{*}:=\left\{u \in B_{R} \mid \forall\right.$ $t \in[0,1], \underline{\omega}(t) \leq u(t) \leq \bar{\omega}(t)\}$.

Proof. From the inequality (23), similar to the proof of Theorem 14 , we can see that for any $t \in[0,1]$,

$$
\begin{aligned}
\bar{\omega}(t) & \leq \bar{\rho}^{q-1} \int_{0}^{1} G_{*}(s, s) \varphi_{q}\left(\int_{0}^{1} H_{*}(\tau, \tau) \tau^{-\sigma_{0}}(1-\tau)^{-\sigma_{1}} d \tau\right) d s \\
& =\bar{\rho}^{q-1}\left(\frac{B\left(1-\sigma_{0}, \beta-\sigma_{1}\right)}{\bar{M}}\right)^{q-1} \leq R .
\end{aligned}
$$

By using the estimation (22), we know that for any $(t, x)$ $\in(0,1) \times[0, R]$,

$$
\begin{aligned}
t^{\sigma_{0}}(1-t)^{\sigma_{1}} k(t, x) & =\min _{x \leq \xi \leq R} t^{\sigma_{0}}(1-t)^{\sigma_{1}} f(t, \xi) \geq \underline{\rho}, \\
t^{\sigma_{0}}(1-t)^{\sigma_{1}} K(t, x) & =\max _{0 \leq \xi \leq x} t^{\sigma_{0}}(1-t)^{\sigma_{1}} f(t, \xi) \leq \bar{\rho} .
\end{aligned}
$$

Since for any $t \in[0,1], 0 \leq \underline{\omega}(t) \leq \bar{\omega}(t) \leq R$, we have

$$
t^{\sigma_{0}}(1-t)^{\sigma_{1}} k(t, \underline{\omega}(t)) \geq \underline{\rho}, t^{\sigma_{0}}(1-t)^{\sigma_{1}} K(t, \bar{\omega}(t)) \leq \bar{\rho} .
$$

In other words, it holds that for any $t \in(0,1)$,

$$
k(t, \underline{\omega}(t)) \geq \underline{\rho} t^{-\sigma_{0}}(1-t)^{-\sigma_{1}}, K(t, \bar{\omega}(t)) \leq \bar{\rho} t^{-\sigma_{0}}(1-t)^{-\sigma_{1}} .
$$

Using the inequalites (24), we obtain

$$
\begin{gathered}
\bar{\omega}(t) \geq \int_{0}^{1} G(t, s) \varphi_{q}\left(\int_{0}^{1} H(s, \tau) \cdot K(\tau, \bar{\omega}(\tau)) d \tau\right) d s, \\
\underline{\omega}(t) \leq \int_{0}^{1} G(t, s) \varphi_{q}\left(\int_{0}^{1} H(s, \tau) \cdot k(\tau, \underline{\omega}(\tau)) d \tau\right) d s .
\end{gathered}
$$

This implies that the functions $\bar{\omega}(t), \underline{\omega}(t)$ are upper and lower solutions of the integral Equation (46) in $B_{R}$, respectively. Therefore, by Lemma 16, the problem (1) has at least one solution in $S^{*}$.

Theorem 18. Assume that the function $f(t, x)$ is nondecreasing in $x$ and the hypotheses (H1)-(H3) are satisfied. Then, the problem (1) has a maximal solution $x_{\max }(t)$ and a minimal solution $x_{\min }(t)$ in $S^{*}$ and the following estimation holds:

$$
\underline{\omega}(t) \leq x_{\min }(t) \leq x_{\max }(t) \leq \bar{\omega}(t) .
$$

Proof. Put $\bar{x}_{0}:=\bar{\omega}, \underline{x}_{0}:=\underline{\omega}$. Theorem 17 shows that the functions $\bar{x}_{0}, \underline{x}_{0}$ are upper and lower solutions of the integral Equation (46). Now, we construct the iterative sequences $\left\{\bar{x}_{n}\right\},\left\{\underline{x}_{n}\right\}$ as follows:

$$
\begin{aligned}
& \bar{x}_{n}(t)=\int_{0}^{1} G(t, s) \varphi_{q}\left(\int_{0}^{1} H(s, \tau) f\left(\tau, \bar{x}_{n-1}(\tau)\right) d \tau\right) d s, \\
& \underline{x}_{n}(t)=\int_{0}^{1} G(t, s) \varphi_{q}\left(\int_{0}^{1} H(s, \tau) f\left(\tau, \underline{x}_{n-1}(\tau)\right) d \tau\right) d s .
\end{aligned}
$$

Since the function $f(t, x)$ is nondecreasing in $x$, we know that if

$$
\underline{x}_{n-1} \leq \underline{x}_{n}, \underline{x}_{n} \leq \bar{x}_{n}, \bar{x}_{n} \leq \bar{x}_{n-1},
$$

then

$$
\underline{x}_{n} \leq \underline{x}_{n+1}, \underline{x}_{n+1} \leq \bar{x}_{n+1}, \bar{x}_{n+1} \leq \bar{x}_{n} .
$$

Considering that the functions $\bar{x}_{0}, \underline{x}_{0}$ are upper and lower solutions of the integral Equation (46), we also have

$$
\underline{x}_{0} \leq \underline{x}_{1}, \underline{x}_{1} \leq \bar{x}_{1}, \bar{x}_{1} \leq \bar{x}_{0} .
$$

So, it is obvious that for any $n \in \mathbf{N}$,

$$
\underline{\omega}=\underline{x}_{0} \leq \underline{x}_{n} \leq \underline{x}_{n+1} \leq \bar{x}_{n+1} \leq \bar{x}_{n} \leq \bar{x}_{0}=\bar{\omega} .
$$

This implies that for the sequences $\left\{\bar{x}_{n}\right\},\left\{\underline{x}_{n}\right\}$, passing to the limit as $n \longrightarrow \infty$, the limits exist. And denoting

$$
x_{\max }=\lim _{n \rightarrow \infty} \bar{x}_{n}, x_{\min }=\lim _{n \rightarrow \infty} \underline{x}_{n},
$$

we can get

$$
\underline{\omega} \leq x_{\min } \leq x_{\max } \leq \bar{\omega} .
$$

Taking the limit $n \longrightarrow \infty$ on both sides of the inequality (25), by the Lebesgue dominated convergence theorem, we obtain

$$
\begin{aligned}
& x_{\min }(t)=\int_{0}^{1} G(t, s) \varphi_{q}\left(\int_{0}^{1} H(s, \tau) f\left(\tau, x_{\min }(\tau)\right) d \tau\right) d s, \\
& x_{\max }(t)=\int_{0}^{1} G(t, s) \varphi_{q}\left(\int_{0}^{1} H(s, \tau) f\left(\tau, x_{\max }(\tau)\right) d \tau\right) d s .
\end{aligned}
$$

Therefore, it holds that the functions $x_{\min }, x_{\max }$ are solutions to the problem (1). 
On the other hand, for any solution to the problem (1) $x \in S^{*}$, it can be easily seen that

$$
\begin{aligned}
\underline{x}_{0} & \leq x \leq \bar{x}_{0}, \\
x(t) & =\int_{0}^{1} G(t, s) \varphi_{q}\left(\int_{0}^{1} H(s, \tau) f(\tau, x(\tau)) d \tau\right) d s, \\
\underline{x}_{1}(t) & =\int_{0}^{1} G(t, s) \varphi_{q}\left(\int_{0}^{1} H(s, \tau) f\left(\tau, \underline{x}_{0}(\tau)\right) d \tau\right) d s, \\
\bar{x}_{1}(t) & =\int_{0}^{1} G(t, s) \varphi_{q}\left(\int_{0}^{1} H(s, \tau) f\left(\tau, \bar{x}_{0}(\tau)\right) d \tau\right) d s .
\end{aligned}
$$

Since the function $f(t, x)$ is nondecreasing in $x$, it is obvious that

$$
\underline{x}_{1} \leq x \leq \bar{x}_{1} .
$$

By induction, we know that for any $n \in \mathbf{N}$,

$$
\underline{x}_{n} \leq x \leq \bar{x}_{n} .
$$

Taking the limit $n \longrightarrow \infty$ on both sides of the inequality (26), we can see

$$
x_{\min } \leq x \leq x_{\max } .
$$

This completes the proof.

Remark 19. If maximal and minimal solutions of the problem (1) exist in $S^{*}$ and they are equal to each other, then (1) has a unique solution in $S^{*}$.

Remark 20. If $\underline{\rho}>0$, then the problem (1) has a positive solution in $S^{*}$.

3.3. The Uniqueness Results for Problem (1). The Gauss hypergeometric function ${ }_{2} F_{1}(a, b ; c ; s)$ is defined in the unit disk as the sum of the hypergeometric series

$$
{ }_{2} F_{1}(a, b ; c ; s)=\sum_{k=0}^{\infty} \frac{(a)_{k}(b)_{k}}{(c)_{k}} \frac{s^{k}}{k !},
$$

where $|z|<1, a, b \in \mathbf{C}, c \in \mathbf{C} \backslash \mathbf{Z}_{0}^{-}$and $(a)_{k}$ is the Pochhammer symbol. And it is known that this function has the following properties (see [35]):

(i) ${ }_{2} F_{1}(a, b ; c ; s)={ }_{2} F_{1}(b, a ; c ; s)$

(ii) If $\operatorname{Re}(c-a-b)>0$, then ${ }_{2} F_{1}(a, b ; c ; 1)=\Gamma(c) \Gamma$ $(c-a-b) / \Gamma(c-a) \Gamma(c-b)$

(iii) $(d / d s)^{n} s^{a+n-1}{ }_{2} F_{1}(a, b ; c ; s)=(a)_{n} s^{a-1}{ }_{2} F_{1}(a+n, b$; $c ; s)$ for any $n \in \mathrm{N}$

(iv) If $0<\operatorname{Re}(b)<\operatorname{Re}(c)$ and $|s|<1$, then
${ }_{2} F_{1}(a, b ; c ; s)=\frac{\Gamma(c)}{\Gamma(b) \Gamma(c-b)} \int_{0}^{1} t^{b-1}(1-t)^{c-b-1}(1-s t)^{-a} d t,|s|<1$.

Denote the function $\chi(s)$ as follows:

$$
\chi(s):={ }_{2} F_{1}\left(1-\sigma_{0}, \sigma_{1} ; \beta+\left(1-\sigma_{0}\right) ; s\right), s \in[0,1] .
$$

Lemma 21. Put

$$
\begin{aligned}
& K_{1}=\frac{\Gamma\left(1-\sigma_{0}\right)}{B \Gamma\left(\beta+\left(1-\sigma_{0}\right)\right)} \sum_{i=1}^{m-2} \zeta_{i} \eta_{i}^{\beta-1}\left[\chi(1)-\eta_{i}^{1-\sigma_{0}} \chi\left(\eta_{i}\right)\right], \\
& K_{2}=\frac{\Gamma\left(1-\sigma_{0}\right)}{\Gamma\left(\beta+\left(1-\sigma_{0}\right)\right)}\left[\chi(1)+\frac{1}{B} \sum_{i=1}^{m-2} \zeta_{i} \eta_{i}^{\beta-1}\left[\chi(1)-\eta_{i}^{1-\sigma_{0}} \chi\left(\eta_{i}\right)\right]\right] .
\end{aligned}
$$

Then, the following estimation holds

$$
K_{1} s^{\beta-1} \leq \int_{0}^{1} H(s, \tau) \tau^{-\sigma_{0}}(1-\tau)^{-\sigma_{1}} d \tau \leq K_{2} s^{\beta-1} .
$$

Proof. To estimate the function $\int_{0}^{1} H(s, \tau) \tau^{-\sigma_{0}}(1-\tau)^{-\sigma_{1}} d \tau$ by using (15), we should calculate the fractional integral of order $\beta$ of the function $s^{-\sigma_{0}}(1-s)^{-\sigma_{1}}$. By the properties (i), (iv) of the Gauss hypergeometric function, we have

$$
\begin{aligned}
& \frac{1}{\Gamma(\beta)} \int_{0}^{s}(s-\tau)^{\beta-1} \tau^{-\sigma_{0}}(1-\tau)^{-\sigma_{1}} d \tau \\
& \quad=\frac{1}{\Gamma(\beta)} s^{\beta-\sigma_{0}} \int_{0}^{1}(1-u)^{\beta-1} u^{-\sigma_{0}}(1-s u)^{-\sigma_{1}} d u \\
& \quad=\frac{1}{\Gamma(\beta)} s^{\beta-\sigma_{0}} \cdot \frac{\Gamma\left(1-\sigma_{0}\right) \Gamma(\beta)}{\Gamma\left(\beta+\left(1-\sigma_{0}\right){ }^{2}\right.} F_{1}\left(\sigma_{1}, 1-\sigma_{0} ; \beta+\left(1-\sigma_{0}\right) ; s\right) \\
& \quad=\frac{\Gamma\left(1-\sigma_{0}\right)}{\Gamma\left(\beta+\left(1-\sigma_{0}\right)\right)} s^{\beta-\sigma_{0}} \chi(s) .
\end{aligned}
$$

So, we obtain

$$
\begin{aligned}
\int_{0}^{1} H(s, \tau) \tau^{-\sigma_{0}}(1-\tau)^{-\sigma_{1}} d \tau \\
=\frac{\Gamma\left(1-\sigma_{0}\right)}{\Gamma\left(\beta+\left(1-\sigma_{0}\right)\right)}\left[-s^{1-\sigma_{0}} \chi(s)\right. \\
\left.\quad-\frac{1}{B} \sum_{i=1}^{m-2} \zeta_{i} \eta_{i}^{\beta-\sigma_{0}} \chi\left(\eta_{i}\right)+\frac{1}{B} \chi(1)\right] \cdot s^{\beta-1} \\
=\frac{\Gamma\left(1-\sigma_{0}\right)}{\Gamma\left(\beta+\left(1-\sigma_{0}\right)\right)}\left[\chi(1)-s^{1-\sigma_{0}} \chi(s)\right. \\
\left.\quad+\frac{1}{B} \sum_{i=1}^{m-2} \zeta_{i} \eta_{i}^{\beta-1}\left(\chi(1)-\eta_{i}^{1-\sigma_{0}} \chi\left(\eta_{i}\right)\right)\right] \cdot s^{\beta-1} .
\end{aligned}
$$


By putting $d(s)=s^{1-\sigma_{0}} \chi(s)$, we can see

$$
\begin{aligned}
\int_{0}^{1} H(s, \tau) \tau^{-\sigma_{0}}(1-\tau)^{-\sigma_{1}} d \tau \\
=\frac{\Gamma\left(1-\sigma_{0}\right)}{\Gamma\left(\beta+\left(1-\sigma_{0}\right)\right)}[d(1)-d(s) \\
\left.\quad+\frac{1}{B} \sum_{i=1}^{m-2} \zeta_{i} \eta_{i}^{\beta-1}\left(d(1)-d\left(\eta_{i}\right)\right)\right] \cdot s^{\beta-1} .
\end{aligned}
$$

The function $d(s)$ is an increasing function on $[0,1]$. In fact, it is obvious that $d(0)=0$ and $d(s) \geq 0$ for any $s \in[0,1]$, and by using the property (iii) of the Gauss hypergeometric function, we can get that for any $s>0$,

$$
d^{\prime}(s)=\left(1-\sigma_{0}\right) s^{-\sigma_{0}}{ }_{2} F_{1}\left(2-\sigma_{0} ; \sigma_{1} ; \beta+\left(1-\sigma_{0}\right) ; s\right)>0
$$

The proof is completed.

From the property (ii) of the Gauss hypergeometric function, we can calculate $\chi(1)$ as

$$
\chi(1)=\frac{\Gamma\left(\beta+\left(1-\sigma_{0}\right)\right) \Gamma\left(\beta-\sigma_{1}\right)}{\Gamma(\beta) \Gamma\left(\beta+1-\sigma_{0}-\sigma_{1}\right)} .
$$

(H4). There exists $L>0$ such that for any $t \in[0,1]$ and any $x_{1}, x_{2} \in[0, R]$,

$$
\left|F\left(t, x_{1}\right)-F\left(t, x_{2}\right)\right| \leq L\left|x_{1}-x_{2}\right| .
$$

Lemma 22. Assume that the hypotheses (H1)-(H4) are satisfied. Then, the followings hold for any $x, y \in B_{R}$ :

(i) If $1<p<2$, then

$$
\|T x-T y\| \leq \frac{(q-1) \bar{\rho}^{q-2} K_{2}^{q-1} L}{A(\alpha-\gamma) \Gamma(\alpha)} \cdot\|x-y\|
$$

(ii) If $p>2$, then

$$
\|T x-T y\| \leq \frac{(q-1) K_{2} L}{A(\alpha-\gamma) \Gamma(\alpha) \underline{\rho}^{2-q} K_{1}^{2-q}} \cdot\|x-y\|
$$

Proof. From the definition of the operator $T$, we can see

$$
\begin{aligned}
|T x(t)-T y(t)| \leq & \int_{0}^{1} G(t, s) \mid \varphi_{q}\left(\int_{0}^{1} H(s, \tau) f(\tau, x(\tau)) d \tau\right) \\
& -\varphi_{q}\left(\int_{0}^{1} H(s, \tau) f(\tau, y(\tau)) d \tau\right) \mid d s .
\end{aligned}
$$

Since $\|x\|,\|y\| \leq R$, by using Lemma 21 and inequality (22), we have

$$
\begin{aligned}
& \underline{\rho} K_{1} s^{\beta-1} \leq \int_{0}^{1} H(s, \tau) f(\tau, x(\tau)) d \tau \leq \bar{\rho} K_{2} s^{\beta-1}, \\
& \underline{\rho} K_{1} s^{\beta-1} \leq \int_{0}^{1} H(s, \tau) f(\tau, y(\tau)) d \tau \leq \bar{\rho} K_{2} s^{\beta-1} .
\end{aligned}
$$

(i) If $1<p<2$, then $q>2$. So, by the inequality (17) and the hypothesis (H4), we obtain that for any $t \in[0,1]$

$$
\begin{aligned}
|T x(t)-T y(t)| \leq & (q-1)\left(\bar{\rho} K_{2}\right)^{q-2} \int_{0}^{1} G(t, s) s^{(\beta-1)(q-2)} \\
& \cdot \int_{0}^{1} H(s, \tau) \tau^{-\sigma_{0}}(1-\tau)^{-\sigma_{1}} \\
& \cdot|F(\tau, x(\tau))-F(\tau, y(\tau))| d \tau d s \\
\leq & (q-1)\left(\bar{\rho} K_{2}\right)^{q-2} L\|x-y\| \int_{0}^{1} G(t, s) s^{(\beta-1)(q-2)} \\
& \cdot \int_{0}^{1} H(s, \tau) \tau^{-\sigma_{0}}(1-\tau)^{-\sigma_{1}} d \tau d s \\
\leq & (q-1) \bar{\rho}^{q-2} K_{2}^{q-1} L\|x-y\| \int_{0}^{1} G(t, s) s^{(\beta-1)(q-1)} d s \\
\leq & (q-1) \bar{\rho}^{q-2} K_{2}^{q-1} L\|x-y\| \int_{0}^{1} G(s, s) d s \\
\leq & \frac{(q-1) \bar{\rho}^{q-2} K_{2}^{q-1} L}{A(\alpha-\gamma) \Gamma(\alpha)} \cdot\|x-y\|
\end{aligned}
$$

(ii) If $p>2$, then $1<q<2$. Employing the inequality (18) and the hypothesis (H4), we have that for any $t \in[0,1]$

$$
\begin{aligned}
|T x(t)-T y(t)| \leq & (q-1)\left(\underline{\rho} K_{1}\right)^{q-2} \int_{0}^{1} G(t, s) s^{(\beta-1)(q-2)} \\
& \cdot \int_{0}^{1} H(s, \tau) \tau^{-\sigma_{0}}(1-\tau)^{-\sigma_{1}} \\
& \cdot|F(\tau, x(\tau))-F(\tau, y(\tau))| d \tau d s \\
\leq & (q-1)\left(\underline{\rho} K_{1}\right)^{q-2} L\|x-y\| \int_{0}^{1} G(t, s) s^{(\beta-1)(q-2)} \\
& \cdot \int_{0}^{1} H(s, \tau) \tau^{-\sigma_{0}}(1-\tau)^{-\sigma_{1}} d \tau d s \\
\leq & (q-1) \underline{\rho^{q-2} K_{1}^{q-2} K_{2} L\|x-y\|} \\
& \cdot \int_{0}^{1} G(t, s) s^{(\beta-1)(q-1)} d s \\
\leq & (q-1) \underline{\rho}^{q-2} K_{1}^{q-2} K_{2} L\|x-y\| \int_{0}^{1} G_{*}(s, s) d s \\
\leq & \frac{(q-1) K_{2} L}{A(\alpha-\gamma) \Gamma(\alpha) \underline{\rho}^{2-q} K_{1}^{2-q}} \cdot\|x-y\|
\end{aligned}
$$


Theorem 23. Assume that the hypotheses (H1)-(H4) are satisfied. Then, the problem (1) has a unique solution in $S^{*}$ if the following conditions hold

(i) If $1<p<2$, then $(q-1) \bar{\rho}^{q-2} K_{2}^{q-1} L / A(\alpha-\gamma) \Gamma(\alpha)<1$

(ii) If $p>2$, then $(q-1) K_{2} L / A(\alpha-\gamma) \Gamma(\alpha) \underline{\rho}^{2-q} K_{1}^{2-q}<1$

Proof. Let $x \neq y$ be two different solutions to the problem (1) in $S^{*}$. Using Lemma 22 we know that if $1<p<2$, then

$$
\begin{gathered}
\|x-y\|=\|T x-T y\| \leq \frac{(q-1) \bar{\rho}^{q-2} K_{2}^{q-1} L}{A(\alpha-\gamma) \Gamma(\alpha)} \\
\cdot\|x-y\|<\|x-y\|,
\end{gathered}
$$

and if $p>2$, then

$$
\begin{gathered}
\|x-y\|=\|T x-T y\| \leq \frac{(q-1) K_{2} L}{A(\alpha-\gamma) \Gamma(\alpha) \underline{\rho}^{2-q} K_{1}^{2-q}} \\
\cdot\|x-y\|<\|x-y\| .
\end{gathered}
$$

This is a contradiction. The proof is completed.

\section{Examples}

The following examples are given to illustrate our main results:

Example 1. Consider the singular four-point boundary value problem

$$
\left\{\begin{array}{l}
D_{0+}^{1.5}\left(\varphi_{3}\left(D_{0+}^{1.75} x(t)\right)\right)=\frac{\ln (5+x(t))}{2 \sqrt{t(1-t)}}, 0<t<1, \\
x(0)=0, D_{0+}^{1.75} x(0)=0 \\
D_{0+}^{0.4} x(1)=0.1 D_{0+}^{0.4} x(0.3)+0.1 D_{0+}^{0.4} x(0.7) \\
\varphi_{3}\left(D_{0+}^{1.75} x(1)\right)=0.1 \varphi_{3}\left(D_{0+}^{1.75} x(0.3)\right)+0.1 \varphi_{3}\left(D_{0+}^{1.75} x(0.7)\right)
\end{array}\right.
$$

The problem (27) can be regarded as the singular boundary value problem (1) where $f(t, x)=\ln (5+x) / 2 \sqrt{t(1-t)}$, $p=3, \alpha=1.75, \beta=1.5, \gamma=0.4, \eta_{1}=0.3, \eta_{2}=0.7, \xi_{1}=0.1, \xi_{2}$ $=0.1, \zeta_{1}=0.1$, and $\zeta_{2}=0.1$. Then, we have

$q=1.5<2, A=0.846122 \cdots, B=0.861562 \cdots, \bar{M}=0.841503 \cdots$

We check that the hypotheses (H1)-(H3) are satisfied. Putting $\sigma_{0}=\sigma_{1}=1 / 2$, it can be easily seen that

$$
\begin{array}{r}
\lim _{t \rightarrow 0+} \sqrt{t} \cdot \frac{\ln (5+x)}{2 \sqrt{t(1-t)}}=\frac{\ln (5+x)}{2}>0, \\
\lim _{t \rightarrow 1-} \sqrt{1-t} \cdot \frac{\ln (5+x)}{2 \sqrt{t(1-t)}}=\frac{\ln (5+x)}{2}>0,
\end{array}
$$

which means that the hypothesis (H1) holds. Put $g(x)=$ $2+(x / 2)$. Then, the function $g(x)$ is nondecreasing in $x$. Since $((\ln (5+x)) / 2) \leq g(x)$, the hypothesis (H2) is satisfied. The hypothesis (H3) can be checked, while for $R=3$,

$$
\begin{aligned}
\frac{R^{p-1}}{g(R)} & =\frac{18}{7}=2.57143 \cdots, \\
\frac{B\left(1-\sigma_{0}, \beta-\sigma_{1}\right)}{\bar{M}} & =2.3767 \cdots
\end{aligned}
$$

The constants $\rho, \bar{\rho}$ are calculated as

$$
\begin{aligned}
& \underline{\rho}=\frac{\ln 5}{2}=0.804719 \cdots, \\
& \bar{\rho}=2+\frac{3}{2}=3.5 .
\end{aligned}
$$

Therefore, from Remark 20, we can know that the boundary value problem (27) has a positive solution.

On the other hand, since the function $f(t, x)=((\ln (5+$ $x)) /(2 \sqrt{t(1-t)}))$ is nondecreasing in $x$, we can construct iterative sequences $\left\{\bar{x}_{n}\right\}_{n=0}^{\infty},\left\{\underline{x}_{n}\right\}_{n=0}^{\infty}$ initialized at

$$
\begin{aligned}
& \bar{x}_{0}(t)=\sqrt{\bar{\rho}} \int_{0}^{1} G(t, s) \varphi_{1.5}\left(\int_{0}^{1} \frac{H(s, \tau)}{\sqrt{\tau(1-\tau)}} d \tau\right) d s, \\
& \underline{x}_{0}(t)=\sqrt{\underline{\rho}} \int_{0}^{1} G(t, s) \varphi_{1.5}\left(\int_{0}^{1} \frac{H(s, \tau)}{\sqrt{\tau(1-\tau)}} d \tau\right) d s,
\end{aligned}
$$

and defined by

$$
\begin{array}{r}
\bar{x}_{n+1}(t)=\int_{0}^{1} G(t, s) \varphi_{1.5}\left(\int_{0}^{1} H(s, \tau) f\left(\tau, \bar{x}_{n}(\tau)\right) d \tau\right) d s, \\
\underline{x}_{n+1}(t)=\int_{0}^{1} G(t, s) \varphi_{1.5}\left(\int_{0}^{1} H(s, \tau) f\left(\tau, \underline{x}_{n}(\tau)\right) d \tau\right) d s, \\
n=0,1,2, \cdots .
\end{array}
$$

Then, the sequences $\left\{\bar{x}_{n}\right\}_{n=0}^{\infty},\left\{\underline{x}_{n}\right\}_{n=0}^{\infty}$ are convergent to the maximal and minimal solutions of the problem (27), respectively.

Putting $L=1 / 10$ and $F(t, x)=((\ln (5+x)) / 2)$, it holds that for any $t \in[0,1]$ and any $x_{1}, x_{2} \geq 0$,

$$
\left|F\left(t, x_{1}\right)-F\left(t, x_{2}\right)\right|<L\left|x_{1}-x_{2}\right|
$$


This implies that the hypothesis (H4) holds. By simple calculation, we can get

$$
\begin{aligned}
& K_{1}=0.136124 \cdots, \\
& K_{2}=2.39288 \cdots .
\end{aligned}
$$

Checking the condition (ii) of Theorem 23, we obtain

$$
\frac{(q-1) K_{2} L}{A(\alpha-\gamma) \Gamma(\alpha) \underline{\rho}^{2-q} K_{1}^{2-q}}=0.344342 \cdots<1 .
$$

Finally, we can find that the boundary value problem (27) has a unique solution and the iterative sequences (27) are convergent to the unique solution of the given problem.

Example 2. Consider the following singular four-point boundary value problem:

$$
\left\{\begin{array}{l}
D_{0+}^{1.5}\left(\varphi_{1.5}\left(D_{0+}^{1.75} x(t)\right)\right)=\frac{\ln (2+x(t))}{10 \sqrt{t(1-t)}}, 0<t<1, \\
x(0)=0, D_{0+}^{1.75} x(0)=0, \\
D_{0+}^{0.4} x(1)=0.1 D_{0+}^{0.4} x(0.3)+0.1 D_{0+}^{0.4} x(0.7), \\
\varphi_{1.5}\left(D_{0+}^{1.75} x(1)\right)=0.1 \varphi_{1.5}\left(D_{0+}^{1.75} x(0.3)\right)+0.1 \varphi_{1.5}\left(D_{0+}^{1.75} x(0.7)\right) .
\end{array}\right.
$$

The problem (30) can be regarded as the boundary value problem (1) where $f(t, x)=\ln (2+x) / 10 \sqrt{t(1-t)}, p=1.5$, $\alpha=1.75, \beta=1.5, \gamma=0.4, \eta_{1}=0.3, \eta_{2}=0.7, \xi_{1}=0.1, \xi_{2}=0.1$, $\zeta_{1}=0.1$, and $\zeta_{2}=0.1$. Then, we can get

$$
\begin{aligned}
q & =3>2, \\
A & =0.846122 \cdots, \\
B & =0.861562 \cdots, \\
M & =0.782325 \cdots .
\end{aligned}
$$

Now, we see whether the hypotheses (H1)-(H3) are satisfied. Putting $\sigma_{0}=\sigma_{1}=1 / 2$, we have

$$
\begin{array}{r}
\lim _{t \rightarrow 0+} \sqrt{t} \cdot \frac{\ln (2+x)}{10 \sqrt{t(1-t)}}=\frac{\ln (2+x)}{10}>0, \\
\lim _{t \rightarrow 1-} \sqrt{1-t} \cdot \frac{\ln (2+x)}{10 \sqrt{t(1-t)}}=\frac{\ln (2+x)}{10}>0,
\end{array}
$$

which means that the hypothesis (H1) holds. Put $g(x)=$ $((1+x) / 10)$. Then, the function $g(x)$ is nondecreasing in $x$. Since $((\ln (2+x)) / 10) \leq g(x)$, the hypothesis (H2) is satisfied. The hypothesis (H3) can be checked, while for $R=1$,

$$
\begin{aligned}
\frac{R^{p-1}}{g(R)} & =5, \\
\frac{B\left(1-\sigma_{0}, \beta-\sigma_{1}\right)}{\bar{M}} & =2.55648 \cdots
\end{aligned}
$$

The constants $\underline{\rho}, \bar{\rho}$ are obtained as

$$
\begin{aligned}
& \underline{\rho}=\frac{\ln 2}{10}=0.0693147 \cdots \\
& \bar{\rho}=\frac{1+1}{10}=0.2 .
\end{aligned}
$$

Therefore, Remark 20 provides that the boundary value problem (30) has a positive solution.

Since the function $f(t, x)=((\ln (2+x)) /(10 \sqrt{t(1-t)}))$ $(/)$ is nondecreasing in $x$, we can construct iterative sequences $\left\{\bar{x}_{n}\right\}_{n=0}^{\infty},\left\{\underline{x}_{n}\right\}_{n=0}^{\infty}$ initialized at

$$
\begin{aligned}
& \bar{x}_{0}(t)=\sqrt{\bar{\rho}} \int_{0}^{1} G(t, \quad s) \varphi_{3}\left(\int_{0}^{1} \frac{H(s, \quad \tau)}{\sqrt{\tau(1-\tau)}} d \tau\right) d s, \\
& \underline{x}_{0}(t)=\sqrt{\underline{\rho}} \int_{0}^{1} G(t, s) \varphi_{3}\left(\int_{0}^{1} \frac{H(s, \tau)}{\sqrt{\tau(1-\tau)}} d \tau\right) d s
\end{aligned}
$$

and defined by

$$
\begin{array}{r}
\bar{x}_{n+1}(t)=\int_{0}^{1} G(t, s) \varphi_{3}\left(\int_{0}^{1} H(s, \tau) f\left(\tau, \bar{x}_{n}(\tau)\right) d \tau\right) d s, \\
\underline{x}_{n+1}(t)=\int_{0}^{1} G(t, s) \varphi_{3}\left(\int_{0}^{1} H(s, \tau) f\left(\tau, \underline{x}_{n}(\tau)\right) d \tau\right) d s, \\
n=0,1,2, \cdots .
\end{array}
$$

Then, the sequences $\left\{\bar{x}_{n}\right\}_{n=0}^{\infty},\left\{\underline{x}_{n}\right\}_{n=0}^{\infty}$ are convergent to the maximal and minimal solutions of the problem (30), respectively.

Putting $L=1 / 20$ and $F(t, x)=((\ln (2+x)) / 10)$, it holds that for any $t \in[0,1]$ and any $x_{1}, x_{2} \geq 0$,

$$
\left|F\left(t, x_{1}\right)-F\left(t, x_{2}\right)\right|<L\left|x_{1}-x_{2}\right|
$$

This assures that the hypothesis (H4) holds. A simple calculation gives

$$
K_{2}=2.39288 \cdots
$$

Checking the condition (i) of Theorem 23, we obtain

$$
\frac{(q-1) \bar{\rho}^{q-2} K_{2}^{q-1} L}{A(\alpha-\gamma) \Gamma(\alpha)}=0.109084 \cdots<1 .
$$

Finally, we can find that the boundary value problem (30) has a unique solution and the iterative sequences (32) are convergent to the unique solution of the given problem.

\section{Conclusion}

In this paper, we have studied the existence and uniqueness results and upper and lower solution methods for multipoint boundary value problems for singular fractional differential 
equations with the $p$-Laplacian operator. For this purpose, we used some fixed-point theorems such as the Leray-Schauder nonlinear alternative and the Schauder fixed-point theorem to prove the existence of positive solutions to the problem (1). And by employing the upper and lower solutions technique, we derived a new approach to obtain the maximal and minimal solutions to the given problem. After that, some useful properties of the Gauss hypergeometric function were applied to the establishment of our uniqueness results. For the application of this work, two expressive examples were illustrated.

\section{Data Availability}

No data were used to support this study.

\section{Conflicts of Interest}

There is no competing interest among the authors regarding the publication of the article.

\section{Authors' Contributions}

All authors carried out the proof and conceived of the study. All authors read and approved the final manuscript.

\section{References}

[1] S. G. Samko, A. A. Kilbas, and O. I. Marichev, Fractional integrals and derivatives: theory and applications, Gordon and Breach, Yverdon, Switzerland, 1993.

[2] I. Podlubny, Fractional differential equations, Academic Press, San Diego, CA, USA, 1999.

[3] L. S. Leibenson, "General problem of the movement of a compressible fluid in a porous medium," Izvestiya Akademii Nauk Kirgizskoĭ SSR, vol. 9, pp. 7-10, 1983.

[4] K. Jong, "Existence and Uniqueness of positive solutions of a kind of multi-point boundary value problems for nonlinear fractional differential equations with $p$-Laplacian operator," Mediterranean Journal of Mathematics, vol. 15, no. 3, 2018.

[5] X. Liu, M. Jia, and X. Xiang, "On the solvability of a fractional differential equation model involving the $p$-Laplacian operator," Computers \& Mathematics with Applications, vol. 64, no. 10, pp. 3267-3275, 2012.

[6] W. Wu and X. Zhou, "Eigenvalue of Fractional Differential Equations with $p$-Laplacian Operator," Discrete Dynamics in Nature and Society, vol. 2013, 8 pages, 2013.

[7] K. Jong, H. Choi, and Y. Ri, "Existence of positive solutions of a class of multi-point boundary value problems for_p_-Laplacian fractional differential equations with singular source terms," Communications in Nonlinear Science and Numerical Simulation, vol. 72, pp. 272-281, 2019.

[8] C. Bai and J. Fang, "The existence of a positive solution for a singular coupled system of nonlinear fractional differential equations," Applied Mathematics and Computation, vol. 150, no. 3, pp. 611-621, 2004.

[9] Z. Bai and T. Qiu, "Existence of positive solution for singular fractional differential equation," Applied Mathematics and Computation, vol. 215, no. 7, pp. 2761-2767, 2009.

[10] W. Feng, S. Sun, Z. Han, and Y. Zhao, "Existence of solutions for a singular system of nonlinear fractional differential equa- tions," Computers \& Mathematics with Applications, vol. 62, no. 3, pp. 1370-1378, 2011.

[11] W. Feng, S. Sun, and Y. Sun, "Existence of positive solutions for a generalized and fractional ordered Thomas-Fermi theory of neutral atoms," Advances in Difference Equations, vol. 2015, no. $1,2015$.

[12] L. Guo, L. Liu, and Y. Wu, "Existence of positive solutions for singular fractional differential equations with infinite-point boundary conditions," Nonlinear Analysis: Modelling and Control, vol. 21, no. 5, pp. 635-650, 2016.

[13] J. Henderson, R. Luca, and A. Tudorache, "On a system of fractional differential equations with coupled integral boundary conditions," Fractional Calculus and Applied Analysis, vol. 18, no. 2, pp. 361-386, 2015.

[14] J. Henderson and R. Luca, "Systems of Riemann-Liouville fractional equations with multi-point boundary conditions," Applied Mathematics and Computation, vol. 309, pp. 303323, 2017.

[15] J. Jiang, W. Liu, and H. Wang, "Positive solutions to singular Dirichlet-type boundary value problems of nonlinear fractional differential equations," Advances in Difference Equations, vol. 2018, no. 1, 2018.

[16] M. Jleli and B. Samet, "On positive solutions for a class of singular nonlinear fractional differential equations," Boundary Value Problems, vol. 2012, no. 1, 2012.

[17] H. Khan, W. Chen, and H. Sun, "Analysis of positive solution and Hyers-Ulam stability for a class of singular fractional differential equations with $p$-Laplacian in Banach space," Mathematical Methods in the Applied Sciences, vol. 41, no. 9, pp. 3430-3440, 2018.

[18] N. Nyamoradi, "Positive solutions for multi-point boundary value problems for nonlinear fractional differential equations," Journal of Contemporary Mathematical Analysis, vol. 48, no. 4, pp. 145-157, 2013.

[19] S. Sun, Y. Zhao, Z. Han, and M. Xu, "Uniqueness of positive solutions for boundary value problems of singular fractional differential equations," Inverse Problems in Science and Engineering, vol. 20, no. 3, pp. 299-309, 2012.

[20] C. Wang, R. Wang, S. Wang, and C. Yang, "Positive solution of singular boundary value problem for a nonlinear fractional differential equation," Boundary Value Problems, vol. 2011, 12 pages, 2011.

[21] S. Vong, "Positive solutions of singular fractional differential equations with integral boundary conditions," Mathematical and Computer Modelling, vol. 57, no. 5-6, pp. 1053-1059, 2013.

[22] H. Wang, L. Zhang, and X. Wang, "New unique existence criteria for higher-order nonlinear singular fractional differential equations," Nonlinear Analysis: Modelling and Control, vol. 24, no. 1, pp. 95-120, 2018.

[23] Y. Wang and L. Liu, "Necessary and sufficient condition for the existence of positive solution to singular fractional differential equations," Advances in Difference Equations, vol. 2015, no. 1, 2015.

[24] Y. Wang, L. Liu, and Y. Wu, "Positive solutions for a class of fractional boundary value problem with changing sign nonlinearity," Nonlinear Analysis: Theory, Methods \& Applications, vol. 74, no. 17, pp. 6434-6441, 2011.

[25] Z. Wei, C. Pang, and Y. Ding, "Positive solutions of singular Caputo fractional differential equations with integral boundary conditions," Communications in Nonlinear Science and Numerical Simulation, vol. 17, no. 8, pp. 3148-3160, 2012. 
[26] S. Xie and Y. Xie, "Positive solutions of a system for nonlinear singular higher-order fractional differential equations with fractional multi-point boundary conditions," Boundary Value Problems, vol. 2016, no. 1, 2016.

[27] X. Zhang, L. Wang, and Q. Sun, "Existence of positive solutions for a class of nonlinear fractional differential equations with integral boundary conditions and a parameter," Applied Mathematics and Computation, vol. 226, pp. 708-718, 2014.

[28] Q. Zhong and X. Zhang, "Positive solution for higher-order singular infinite-point fractional differential equation with p-Laplacian," Advances in Difference Equations, vol. 2016, no. 1, 2016.

[29] W.-X. Zhou, Y.-D. Chu, and D. Băleanu, "Uniqueness and existence of positive solutions for a multi-point boundary value problem of singular fractional differential equations," Advances in Difference Equations, vol. 2013, no. 1, 2013.

[30] A. Petruşel and I. A. Rus, "Fixed point theory in terms of a metric and of an order relation," Fixed Point Theory, vol. 20, no. 2, pp. 601-622, 2019.

[31] Z. Bai and H. Lu, "Positive solutions for boundary value problem of nonlinear fractional differential equation," Journal of Mathematical Analysis and Applications, vol. 311, no. 2, pp. 495-505, 2005.

[32] E. Zeidler, Nonlinear Functional Analysis and its Applications, Springer New York, New York, NY, 1986.

[33] A. Granas and J. Dugundji, Fixed Point Theory, Springer New York, New York, NY, 2003.

[34] Z.-W. Lv, "Positive solutions of $m$-point boundary value problems for fractional differential equations," Advances in Difference Equations, vol. 2011, Article ID 571804, 13 pages, 2011.

[35] A. A. Kilbas, H. M. Srivastava, and J. J. Trujillo, Theory and Applications of Fractional Differential Equations, Elsevier, Amsterdam, The Netherlands, 2006. 